\title{
Nuclear Quantum Effects on Autoionization of Water Isotopologues Studied by Ab Initio Path Integral Molecular Dynamics
}

Bo Thomsen ${ }^{1, a)}$ and Motoyuki Shiga ${ }^{1, b)}$

CCSE, Japan Atomic Energy Agency, 178-4-4, Wakashiba, Kashiwa, Chiba, 277-0871, Japan

(Dated: 1 February 2021)

In this study we investigate the nuclear quantum effects (NQEs) on the acidity constant $\left(\mathrm{p} K_{A}\right)$ of liquid water isotopologues at the ambient condition by path integral molecular dynamics (PIMD) simulations. We compared simulations using a fully explicit solvent model with a classical polarizable force field, density functional tight binding, and ab initio density functional theory, which correspond to empirical, semiempirical, and ab initio PIMD simulations, respectively. The centroid variable with respect to the proton coordination number of a water molecule was restrained to compute the gradient of the free energy, which measures the reversible work of the proton abstraction for the quantum mechanical system. The free energy curve obtained by thermodynamic integration was used to compute the $\mathrm{p} K_{A}$ value based on probabilistic determination. This technique not only reproduces the $\mathrm{p} K_{A}$ value of liquid $\mathrm{D}_{2} \mathrm{O}$ experimentally measured (14.86) but also allows for a theoretical prediction of the $\mathrm{p} K_{A}$ values of liquid $\mathrm{T}_{2} \mathrm{O}$, aqueous HDO and HTO which are unknown due to its scarcity. It is also shown that the NQEs on the free energy curve can result in a downshift of $4.5 \pm 0.9 \mathrm{p} K_{A}$ units in the case of liquid water, which indicates that the NQEs plays an indispensable role in the absolute determination of $\mathrm{p} K_{A}$. The results of this study can help to inform further extensions into the calculation of the acidity constants of isotope substituted species with high accuracy.

\footnotetext{
a)Electronic mail: thomsen.bo@jaea.go.jp

b)Electronic mail: shiga.motoyuki@jaea.go.jp
} 


\section{INTRODUCTION}

The acidity constant, $\mathrm{p} K_{A}$, plays a fundamental role in acid-base chemistry. There is no doubt about the importance of quantitatively estimating $\mathrm{p} K_{A}$ for a given functional group in a molecular species. Computational chemistry rooted in molecular theory has the merit that it is possible to evaluate $\mathrm{p} K_{A}$ independent of experiments. ${ }^{1 / 3}$ However, computational evaluation of $\mathrm{p} K_{A}$ has yet to reach the quantitative level of accuracy, even for the most basic case of liquid water, i.e., the water autoionization constant, $\mathrm{p} K_{W}$.

Previous computational evaluations of $\mathrm{p} K_{A}$ have been based on either an implicit solvent model, in which a solute molecule is embedded in a solvent described by polarizable continuum medium,,$\frac{13}{13}$ or an explicit solvent model, in which both the solute and the solvent

are described explicitly as molecules. $14 \sqrt[22]{2}$ In general, the implicit solvent model is able to provide $\mathrm{p} K_{A}$ values with small computational effort but limited accuracy. The intrinsic error of the implicit solvent model arises from the lack of complexity to describe solute-solvent interactions.

The inclusion of explicit solvent molecules $23 \sqrt{27}$ and extended conformational sampling 2 have recently been shown to partially solve these issues. Both methods, however, require careful (re)-parametrization of the implicit solvent model and selection of the conformers to be considered in the calculation. It is therefore preferable to deal with the solvent molecules explicitly for quantitatively estimating $\mathrm{p} K_{A}$, where solute-solvent interactions are taken into account properly. In this case $\mathrm{p} K_{A}$ is estimated directly from the free energy change upon the protonation of a solute molecule by molecular dynamics (MD) techniques, such as coordination-constrained MD (often to referred to as the blue-moon ensemble method) $\underline{29 \mid 30}$

$\mathrm{p} K_{A}$ is known to have a strong dependence on thermodynamic conditions such as the temperature and pressure. For liquid water under a pressure of $15 \mathrm{MPa}$, the $\mathrm{p} K_{W}$ value of 14 at the ambient temperature decreases to about 11 in subcritical conditions at $300{ }^{\circ} \mathrm{C}$, and then increases to about 20 in supercritical conditions at $400{ }^{\circ} \mathrm{C}$. ${ }^{31}$ It is also known that $\mathrm{p} K_{A}$ clearly differs between the hydrogen isotopologues, $\frac{32}{2}$ e.g., $\mathrm{p} K_{W}$ of $\mathrm{D}_{2} \mathrm{O}$ under ambient conditions is 14.86 , which is larger than that of $\mathrm{H}_{2} \mathrm{O}, 14.00 .33$ Constrained MD simulations allow the estimation of $\mathrm{p} K_{A}$ under different thermodynamic conditions. However, they will produce identical results for the hydrogen isotopologues, because the free energy change calculated by classical MD does not depend on the nuclear masses. The isotope effect on 
the free energy can be traced back to the quantum nature whereby the kinetic and potential energy operators in the Boltzmann density do not commute. Therefore, it follows that the nuclear quantum effects (NQEs) should not be ignored for the quantitative estimation of $\mathrm{p} K_{A}$. In fact, hydrogen is generally known to exhibit quantum behaviors such as zero-point vibration and tunneling because of its light mass.

The $\mathrm{p} K_{W}$ of water has been a long-standing interest for theoretical studies, since the long time-scale on which the autodissociation takes place makes it difficult to sample efficiently. Furthermore the strong solvation effects of the produced $\mathrm{OH}^{-}$and $\mathrm{H}^{+}$ions make it important to consider the solvation structure explicitly.

The computation of $\mathrm{p} K_{W}$ of liquid water has been done with explicit solvent in several studies. ${ }^{34} \sqrt[37]{37}$ The reaction mechanism and kinetics of the autoionization of liquid water have been the subject in extensive studies ${ }^{[38}[40$ However, none of these studies have explored the NQEs on the $\mathrm{p} K_{W}$ of water in ab initio simulations based on first principles.

In this paper we introduce a coordination-restrained path integral molecular dynamics (PIMD) method based on an explicit solvent model to study the $\mathrm{p} K_{W}$ of liquid water and its isotopologues. PIMD is a rigorous approach that takes account of both nuclear quantum and temperature effects based on the imaginary-time path integral theory for quantum statistical mechanics ${ }^{41}$ 45. PIMD has been used to study the NQEs on several properties of water as summarized in a recent review. $\underline{46}$

In this review the NQEs on the $\mathrm{p} K_{W}$ of water is estimated by extrapolating the effect on the water isotopologues to the case of infinite mass nuclei, which corresponds to the result of a classical MD simulation. The change from classical to quantum nuclei was found to cause a downshift of around $3 \mathrm{p} K_{A}$ units. This empirical shift was subsequently used in the study by Wang et al. to correct the $\mathrm{p} K_{W}$ calculated from classical MD ${ }^{37}$

Two important references in the context of this paper are the study on the solvated proton and hydroxide ion by Marx et al. .47 and the recent study on the NQEs in proton transport in water under an electrical field by Cassone. ${ }^{48}$ Here we will extend the PIMD method for $\mathrm{p} K_{W} / \mathrm{p} K_{A}$ estimations, allowing the computations of quantum free energies upon the protonation of the solute, by restraining the centroid variable of the coordination number $(\mathrm{CN})$.

In Section II we will outline the theory of coordination-restrained PIMD and the calculation of $\mathrm{p} K_{W}$ in terms of probabilistic and absolute methods. Section III contains the 
computational details for the simulations conducted in this study. The results of MD and PIMD will then be discussed in Section IV. Furthermore we will investigate the isotope effects in pure $\mathrm{D}_{2} \mathrm{O}$ and $\mathrm{T}_{2} \mathrm{O}$, and the solvated $\mathrm{HDO}$ and $\mathrm{HTO}$ isotope substituted water like molecules. Finally in Section V we will summarize our findings.

\section{THEORY}

\section{A. Coordination Number Restrained Path Integral Molecular Dynamics}

Consider the quantum Hamiltonian for an $N$ atom system within the Born Oppenheimer approximation

$$
\hat{H}=\sum_{I=1}^{N} \frac{\hat{\mathbf{P}}_{I}^{2}}{2 M_{I}}+V\left(\hat{\mathbf{R}}_{1}, \ldots, \hat{\mathbf{R}}_{N}\right)
$$

where $M_{I}$ is the mass of the $I$ th particle, $\hat{\mathbf{P}}_{I}^{2}$ is the momentum operator $\left(\hat{P}_{I, x}, \hat{P}_{I, y}, \hat{P}_{I, z}\right)$, $\hat{\mathbf{R}}_{I}$ is the position operator $\left(\hat{R}_{I, x}, \hat{R}_{I, y}, \hat{R}_{I, z}\right)$ and $V$ is the potential. The quantum partition function for this system is given as

$$
Z=\int d \mathbf{R}_{1} \cdots \int d \mathbf{R}_{N}\left\langle\mathbf{R}_{1} \cdots \mathbf{R}_{N}|\exp (-\beta \hat{H})| \mathbf{R}_{1} \cdots \mathbf{R}_{N}\right\rangle=\operatorname{Tr} \exp (-\beta \hat{H})
$$

Here $\beta=\frac{1}{k_{b} T}$, where $k_{b}$ is the Boltzmann constant and $T$ is the temperature. It is assumed that the thermal de Broglie wavelength is smaller than the separation of two identical atoms, thereby allowing us to ignore the possibility for exchange of fermion/boson positions.

By dividing the Boltzmann operator in Equation (2) into $P$ terms, inserting closure relations in coordinate spaces between each term of the Boltzmann operator, and applying the second-order Suzuki-Trotter expansion one can derive the following expression for the partition function,

$$
Z=\lim _{P \rightarrow \infty} Z_{P}
$$

where

$$
\begin{aligned}
Z_{P}= & \prod_{I=1}^{N}\left[\left(\frac{M_{I} P}{2 \pi \beta \hbar^{2}}\right)^{\frac{3 P}{2}} \int d \mathbf{R}_{I}^{(1)} \int d \mathbf{R}_{I}^{(2)} \cdots \int d \mathbf{R}_{I}^{(P)}\right] \\
& \times \exp \left[-\beta\left\{\sum_{s=1}^{P} \sum_{I=1}^{N} \frac{M_{I}}{2} \omega_{P}^{2}\left(\mathbf{R}_{I}^{(s)}-\mathbf{R}_{I}^{(s-1)}\right)^{2}+\sum_{s=1}^{P} \frac{1}{P} V\left(\mathbf{R}_{1}^{(s)}, \ldots, \mathbf{R}_{N}^{(s)}\right)\right\}\right]
\end{aligned}
$$


The constant $\omega_{P}$ is given as $\frac{\sqrt{P}}{\beta \hbar}$. The factor $\prod_{I=1}^{N}\left(\frac{M_{I} P}{2 \pi \beta \hbar^{2}}\right)^{\frac{3 P}{2}}$ is a constant and will be omitted in the following, as it does not alter the relative free energy differences. Rearranging the exponent in the above equation we arrive at the following,

$$
Z_{P} \propto \prod_{I=1}^{N}\left[\int d \mathbf{R}_{I}^{(1)} \int d \mathbf{R}_{I}^{(2)} \cdots \int d \mathbf{R}_{I}^{(P)}\right] \exp \left(-\beta V_{\mathrm{eff}}(\{\mathbf{R}\})\right)
$$

where

$$
V_{\text {eff }}(\{\mathbf{R}\})=\sum_{s=1}^{P}\left\{\sum_{I=1}^{N} \frac{M_{I}}{2} \omega_{P}^{2}\left(\mathbf{R}_{I}^{(s)}-\mathbf{R}_{I}^{(s-1)}\right)^{2}+\frac{1}{P} V\left(\mathbf{R}_{1}^{(s)}, \ldots, \mathbf{R}_{N}^{(s)}\right)\right\}
$$

These two equations show that the quantum behaviour of an $N$ particle system can be mimicked by considering an NP particle system. The individual terms in $P$ are often referred to as beads on a chain, where each bead corresponds to a single copy of the classical system. This system is evolved according to its classical forces and its coupling to a polymer chain. The cost of this method thus scales steeply with the number of beads, and by extension accuracy desired. Convergence with respect to number of beads does however scale inversely with temperature, i.e. a few tens of beads can be sufficient to accurately describe quantum effects for systems under standard conditions.

The blue-moon sampling approach $\frac{29 \mid 30}{13}$ is here used to compute the free energy curve of water autoionization. Following a study by Sprik $\frac{34}{34}$, the $\mathrm{CN}$ of an oxygen atom (labeled as " $\mathrm{O}^{* ")}$ ) is used for studying this reaction. We introduce a rational function for the $\mathrm{CN}$ as

$$
n_{\mathrm{O} *}(\{\mathbf{R}\})=\sum_{j \in H} \frac{1-\left(\frac{r_{\mathrm{O}_{j} j}}{d_{\mathrm{OH}}}\right)^{6}}{1-\left(\frac{r_{\mathrm{O}_{j}}}{d_{\mathrm{OH}}}\right)^{12}},
$$

where $\{\mathbf{R}\}$ is the set of atomic coordinates of the system, $r_{i j}$ are the distances to all hydrogen atoms in the system, and $d_{\mathrm{OH}}$ is a constant set to $1.35 \AA$.

The $\mathrm{CN}$ is in this study is restrained to vary the coordination of hydrogen in a single $\mathrm{OH}^{-}$moiety from one to zero. These conditions correspond to the moiety forming a $\mathrm{H}_{2} \mathrm{O}$ molecule and a solvated $\mathrm{OH}^{-}$ion respectively. In practice, for a random oxygen atom, one of its attached hydrogens is chosen to remain bound, while the other attached hydrogen and all other hydrogens are subject to the $\mathrm{CN}$ restraint. The same method is used in the case of $\mathrm{D}_{2} \mathrm{O}$ and $\mathrm{T}_{2} \mathrm{O}$ for the deuterium and tritium atoms in the simulation, respectively. For HDO and HTO in solution all hydrogens are restrained, while the core $\mathrm{OD}^{-}$or $\mathrm{OT}^{-}$is kept unrestrained. 
To derive the free energy difference in the blue-moon ensemble one has to consider the free energy of a system restrained to a fixed coordination number $\bar{n}_{\mathrm{O}^{*}}$,

$$
A\left(\bar{n}_{\mathrm{O}^{*}}\right)=-\beta^{-1} \log \rho\left(\bar{n}_{\mathrm{O}^{*}}\right)+A_{0} .
$$

$A_{0}$ is a constant term taking care of the constants dropped in Equation (5). This term is only necessary to consider when calculating the absolute value of the free energy, as it will cancel for relative free energy differences. The distribution $\rho\left(\bar{n}_{\mathrm{O}^{*}}\right)$ is the scaled probability for finding the system with the given coordination number $\bar{n}_{O^{*}}$, which is expressed as

$$
\begin{aligned}
& \rho\left(\bar{n}_{\mathrm{O}^{*}}\right)=\left\langle\delta\left(\bar{n}_{\mathrm{O}^{*}}-\frac{1}{P} \sum_{s=1}^{P} n_{\mathrm{O}^{*}}\left(\mathbf{R}^{(s)}\right)\right)\right\rangle \\
= & \lim _{P \rightarrow \infty} Z_{P}^{-1} \prod_{I=1}^{N}\left[\int d \mathbf{R}_{I}^{(1)} \cdots \int d \mathbf{R}_{I}^{(P)}\right] \delta\left(\bar{n}_{\mathrm{O}^{*}}-\frac{1}{P} \sum_{s=1}^{P} n_{\mathrm{O}^{*}}\left(\mathbf{R}^{(s)}\right)\right) \exp \left(-\beta V_{\mathrm{eff}}(\{\mathbf{R}\})\right)
\end{aligned}
$$

in the PIMD formalism. The brackets indicate the ensemble average of the contained function, which is equal to its time average assuming ergodicity. To further simplify the expression a narrow peaked Gaussian function, with an inverse variance given as $\frac{\kappa}{2}$, is used to approximate the delta function, giving

$$
\rho\left(\bar{n}_{\mathrm{O}^{*}}\right) \approx \lim _{P \rightarrow \infty} Z_{P}^{-1} \sqrt{\frac{\beta \kappa}{2 \pi}} \prod_{I=1}^{N}\left[\int d \mathbf{R}_{I}^{(1)} \cdots \int d \mathbf{R}_{I}^{(P)}\right] \exp \left(-\beta V_{\mathrm{eff}}^{\mathrm{cons}}\left(\{\mathbf{R}\}, \bar{n}_{\mathrm{O}^{*}}\right)\right)
$$

where

$$
V_{\mathrm{eff}}^{\text {cons }}\left(\{\mathbf{R}\}, \bar{n}_{\mathrm{O}^{*}}\right)=V_{\mathrm{eff}}(\{\mathbf{R}\})+\frac{\kappa}{2}\left(\bar{n}_{\mathrm{O}^{*}}-\frac{1}{P} \sum_{s=1}^{P} n_{\mathrm{O}^{*}}\left(\mathbf{R}^{(s)}\right)\right)^{2} .
$$

It is exceedingly difficult to calculate the free energy value itself through the above equations. Calculating the derivative of the free energy with respect to the restrained value is, however, less of a challenge. This derivative is given as

$$
f\left(\bar{n}_{\mathrm{O}^{*}}\right)=\frac{\partial A\left(\bar{n}_{\mathrm{O}^{*}}\right)}{\partial \bar{n}_{\mathrm{O}^{*}}}=\left\langle\kappa\left(\bar{n}_{\mathrm{O}^{*}}-\frac{1}{P} \sum_{s=1}^{P} n_{\mathrm{O}^{*}}\left(\mathbf{R}^{(s)}\right)\right)\right\rangle_{\mathrm{eff}},
$$

where the subscript "eff" stands for the sampling by a PIMD simulation with the restraint. This derivative corresponds to the time-averaged force which will be used in the following to calculate the free energy surface as a function of the $\mathrm{CN}$. 


\section{B. Methodology for Calculating the Autoionization Constant of Water}

Using the time-averaged forces, $f\left(\bar{n}_{\mathrm{O}^{*}}\right)$, from Equation 12$)$ and the time-averaged CNs, $n_{\mathrm{O}^{*} i}$, obtained from a number of $\mathrm{CN}$ restrained simulations it is possible to estimate the free energy difference through thermodynamic integration,

$$
\Delta A\left(\bar{n}_{\mathrm{O}^{*} i}\right)=\int_{\bar{n}_{\mathrm{O}^{*}}}^{\bar{n}_{\mathrm{O}^{*} i}} f(\bar{n}) d \bar{n} .
$$

The numerical integral is evaluated by spline interpolation between each of the simulated restrained CNs $\left(\bar{n}_{\mathrm{O}^{*} 1}, \ldots, \bar{n}_{\mathrm{O}^{*} M}\right)$. The numerical integral is here calculated using a third order B-spline which passes through all the calculated points.

Using the free energy surface one can employ a probabilistic (PROB) method to calculate the $\mathrm{p} K_{W}$ of water and $\mathrm{p} K_{A}$ of an acid, as suggested by Davies et al.,17] based on the work of Chandler ${ }^{49}$ This method relies on the relative probability of finding the system in a bound state. For this purpose we define a cutoff bond distance, $R_{c}$, at which the $\mathrm{O}-\mathrm{H}$ bond breaks and the $\mathrm{OH}^{-}$and $\mathrm{H}_{3} \mathrm{O}^{+}$ions are formed. The probability ratio between the bound and dissociated states is given by

$$
\gamma\left(R_{c}\right)=\frac{\int_{0}^{R_{c}} \exp \left(-\beta \Delta A\left(\bar{n}_{\mathrm{O}^{*}}(r)\right)\right) 4 \pi r^{2} d r}{\int_{0}^{R_{\max }} \exp \left(-\beta \Delta A\left(\bar{n}_{\mathrm{O}^{*}}(r)\right)\right) 4 \pi r^{2} d r},
$$

where the factor $4 \pi r^{2}$ arises from the Jacobian of polar coodinates. The mapping $\bar{n}_{\mathrm{O}^{*}}(r)$ is

carried out by assigning the time averaged closest distance between the central oxygen and the restrained hydrogens, $r_{i}$, for the given restraint $\bar{n}_{\mathrm{O}^{*} i}$. The assigned free energy difference calculated in Equation $(13), \Delta A\left(\bar{n}_{\mathrm{O}^{*}}(r)\right)$, can then be linearly interpolated to numerically evaluate the probability. $R_{\max }$ is the time averaged distance when the CN restraint is set to the lowest coordination number, $\bar{n}_{\mathrm{O}^{*} M}$.

The value of $R_{c}$ is determined so that it gives $\mathrm{p} K_{W}=14.00$ for liquid $\mathrm{H}_{2} \mathrm{O}$. For water autoionization

$$
\mathrm{H}_{2} \mathrm{O}(\mathrm{l}) \rightleftharpoons \mathrm{OH}^{-}(\mathrm{aq})+\mathrm{H}^{+}(\mathrm{aq}),
$$

the autoionization constant is

$$
\mathrm{p} K_{W}=-\log \left(\left[\mathrm{OH}^{-}(\mathrm{aq})\right]\left[\mathrm{H}^{+}(\mathrm{aq})\right]\right) .
$$

Rewriting this using the probabilities of water dissociation $\left(\gamma_{W}\left(R_{c}\right)\right)$ from Equation (14) leads to

$$
\mathrm{p} K_{W}\left(R_{c}\right)=-2 \log \left(\left(1-\gamma_{W}\left(R_{c}\right)\right) \frac{N_{W}}{c_{0} V}\right),
$$


where $N_{W}$ and $V$ are the number of water molecules and the volume of the simulation box, respectively, and $c_{0}$ is the standard concentration $(1 \mathrm{M})$. To calculate $R_{c}$, the reference value at standard conditions is used, resulting in

$$
\gamma_{W}\left(R_{c}\right)=1-\frac{10^{-7}}{c_{W}}
$$

where $c_{W}=\frac{N_{W}}{c_{0} V}$ (which is about 55.6 in ambient conditions). This can then be used to find $R_{c}$ for Equation (14). It is then commonly assumed that this distance is also applicable to breaking the $\mathrm{A}-\mathrm{H}$ bond to form $\mathrm{A}^{-}$and $\mathrm{H}_{3} \mathrm{O}^{+}$for a common acid $\mathrm{A}$.

The $\mathrm{p} K_{A}$ of an acid, or acid group of a molecule, $(\mathrm{A})$ in water can be found by considering

$$
\mathrm{AH}(\mathrm{aq}) \rightleftharpoons \mathrm{A}^{-}(\mathrm{aq})+\mathrm{H}^{+}(\mathrm{aq})
$$

and the following expression for the acidity constant $\left(\mathrm{p} K_{A}\right)$,

$$
\mathrm{p} K_{A}=-\log \left(\frac{\left[\mathrm{A}^{-}\right]\left[\mathrm{H}^{+}\right]}{[\mathrm{AH}]}\right) .
$$

Here we have assumed a dilute aqueous solution, where the activities of all species are determined by their concentration in the solution. The solvated protons, $\mathrm{H}^{+}$, can in principle stem from both water autoionization and the dissociation from A leaving us with the following expression for their concentration

$$
\left[\mathrm{H}^{+}\right]=\left[\mathrm{H}^{+}\right]_{\mathrm{acid}}+\left[\mathrm{H}^{+}\right]_{\text {water }}=\left(1-\gamma_{A}\left(R_{c}\right)\right) c_{A}+\left(1-\gamma_{W}\left(R_{c}\right)\right) c_{W}^{\prime}
$$

where $c_{W}^{\prime}=\frac{N_{W}^{\prime}}{c_{0} V}$ and $c_{A}=\frac{N_{A}}{c_{0} V} . N_{W}^{\prime}=N_{W}-N_{A}$, and $N_{A}$ is the number of acid molecules replacing water molecules in the box. It is implicitly assumed that the $\mathrm{p} K_{W}$ of water is unchanged in the solution of $\mathrm{AH}$, a reasonable assumption given that the solution is dilute. Using the probabilities of dissociation for the acid and water we can rewrite Equation (20) as

$$
\begin{aligned}
\mathrm{p} K_{A}(\mathrm{PROB}) & =-\log \left(\frac{\left(1-\gamma_{A}\left(R_{c}\right)\right) c_{A}\left(\left(1-\gamma_{A}\left(R_{c}\right)\right) c_{A}+\left(1-\gamma_{W}\left(R_{c}\right)\right) c_{W}^{\prime}\right)}{c_{A} \gamma_{A}}\right) \\
& =-\log \left(\frac{\left(1-\gamma_{A}\right)}{\gamma_{A}}\left(\frac{\left(1-\gamma_{A}\right) N_{A}}{c_{0} V}+10^{-7} \frac{N_{W}-N_{A}}{N_{W}}\right)\right) .
\end{aligned}
$$

Generally one would find that $\left(1-\gamma_{A}\left(R_{c}\right)\right) \gg\left(1-\gamma_{W}\left(R_{c}\right)\right)$, so the above equation can then be reasonably approximated as

$$
\mathrm{p} K_{A}(\mathrm{PROB}) \approx-\log \left(\frac{\left(1-\gamma\left(R_{c}\right)\right)^{2}}{\left(\gamma\left(R_{c}\right)\right)} \frac{N_{A}}{c_{0} V}\right) .
$$


Equation (23) was used in previous studies to predict the $K_{A}$ of acidic substances where the proton concentration are mainly from the solute dissociation. ${ }^{14}$ In this study we will resort to using Equation (22), since the $\mathrm{p} K_{A}$ of HDO and HTO in aqueous solution is expected to be close to that of the solvent $\mathrm{H}_{2} \mathrm{O}$ itself. This approach is however general to all very weak acids, with $\mathrm{p} K_{A}$ values around their solvent. These types of acids have not previously been studied in this context, which is why most studies have relied on Equation (23) for calculating $\mathrm{p} K_{A}$ values.

Another way of calculating the probabilities used in the equations above is to employ a basic two state model. That is, we assume that the minimum of the free energy surface corresponds to the free energy of the bound state of water and the free energy of the lowest CN restraint corresponds to the dissociated state. From this model we can formulate the probability of finding a water molecule in a dissociated state as

$$
1-\gamma=\frac{\exp (-\beta \Delta A)}{1+\exp (-\beta \Delta A)}
$$

where $\Delta A$ is taken as the difference between the maximum (dissociated state) value and the minimum (bound state) value of $\Delta A\left(\bar{n}_{\mathrm{O}^{*} i}\right)$ in Equation $(13)$. For the autoionization of water and its isotopologues we can approximate this probability as $\exp (-\beta \Delta A)$, as the free energy difference is very large. Inserting this into Equation (17) results in

$$
\mathrm{p} K_{W}(\mathrm{ABS})=-2 \log \left(\exp (-\beta \Delta A) \frac{N_{W}}{c_{0} V}\right)=\frac{2 \beta \Delta A}{\ln (10)}-2 \log \left(\frac{N_{W}}{c_{0} V}\right) .
$$

This method allows us to compare the calculated $\mathrm{p} K_{W}$ of $\mathrm{H}_{2} \mathrm{O}$ to that of $\mathrm{D}_{2} \mathrm{O}$ and $\mathrm{T}_{2} \mathrm{O}$, as opposed to the probabilistic method, in which the $\mathrm{H}_{2} \mathrm{O}$ simulation is used as a reference. In the following this method will be referred to as the absolute (ABS) method, as it depends on the absolute free energy difference between two states.

\section{COMPUTATIONAL DETAILS}

MD and PIMD simulations of liquid $\mathrm{H}_{2} \mathrm{O}$ and its isotopologues were undertaken in the canonical ensemble at temperature $300 \mathrm{~K}$. The interatomic potential and the associated force were computed on the fly by ab initio DFT, semiempirical DFTB, or empirical OSS2 methods. The simulation conditions are listed in Table I. Systems of 32-64 water molecules were contained in cubic boxes with periodic boundary conditions. The box size was set 
such that the number density was 29.86 molecules $/ \AA^{3}$, which amounts to $1.00 \mathrm{~g} / \mathrm{cm}^{3}$ for $\mathrm{H}_{2} \mathrm{O}$. An example structure is depicted in Figure 1 along with snapshots of the constrained water molecule and its surroundings under different CN restraints recorded from the DFT trajectories.

The numerical integration schemes for MD and PIMD were based on the reversible reference system propagation algorithm (RESPA) as implemented in the PIMD software package. $\underline{50}$ The temperature was strongly controlled by attaching massive Nosé-Hoover chain (MNHC) thermostats 5153 to each degree of freedom in the MD simulations. The MNHC thermostats were also attached to each normal mode representing the ring polymer in the PIMD simulations. The fifth-order Suzuki-Yoshida factorization was used for the numerical integration of the MNHC thermostats. The simulations were run for $12.5-25.0$ ps with the step size of $0.25-0.43$ fs depending on the system and the interatomic potential. The restraints with a force constant $\kappa=4-10$ hartree were applied at 15 points in the range $0.16 \leq n_{i} \leq 0.98$. This resulted in a fluctuation of $\mathrm{CN}$ within the order of 0.01 . To deal with the fast oscillation caused by the restraints, the restraint force was updated 5 times per MD/PIMD step in the RESPA technique.

The ab initio DFT energy calculations were carried out using the Vienna ab initio simulation package (VASP) $\sqrt[54]{56}$ We employed the Perdew-Burke-Ernzerhof (PBE) $)^{\sqrt[57]{5}}$ exchange correlation functional and Grimme's D3 van der Waals correction. ${ }^{58}$ The core electrons were taken into account using the projector augmented wave (PAW) method. The valence electrons are expressed in terms of a linear combination of plane wave basis functions with a cutoff at $400 \mathrm{eV}$. Only the $\Gamma$-point of the Brillouin zone was computed.

The semiempirical DFTB energy calculations were carried out using DFTB+ ${ }^{59}$ We employed the third-order self-consistent-charge density-functional tight-binding (SCC-DFTB) ${ }^{60}$ method with the 3ob Slater-Koster parameter set.61

The empirical energy calculations based on the OSS2 ${ }^{62}$ potential were implemented in an in-house version of the PIMD software package. The OSS2 potential can be categorized as a polarizable force field composed of short-range intramolecular bonds and long-range interactions between point charges and induced point dipoles with damping. The parameters in the OSS2 potential are fitted so as to reproduce the ab initio energies of neutral and protonated water clusters at the level of second-order Møller-Plesset perturbation theory (MP2). The OSS2 potential was originally developed for small water clusters in the free 
boundary condition, but it can be applied to bulk water by adaption for periodic boundary conditions. We used the Ewald sum of point charges and induced point dipoles for this, where the point dipoles in the electrostatic field were determined by the matrix inversion method $[6]$

The resulting restraints, forces and trajectories were analysed using a locally developed python script, where the MDAnalysis library ${ }^{6465}$ was used for calculating the O-H distances. The errors reported here were calculated by the method outlined by Flyvbjerg and Petersen. ${ }^{66}$ The errors of the cut off distance $R_{c}$ and the probabilistic $\mathrm{p} K_{A}$ or $\mathrm{p} K_{W}$ are not considered to be correlated, i.e., the errors in $\mathrm{p} K_{W}$ or $\mathrm{p} K_{A}$ are calculated using a fixed value of $R_{c}$. All figures depicting the molecular systems were visualized using the VMD software. $\frac{67}{6}$ The numerical integrals needed for the calculations outlined in the theory section were all carried out using linear interpolation and a step size of $1 \cdot 10^{-4}$ in both $\mathrm{CN}$ and $\mathrm{O}-\mathrm{H}$ distance space.

\section{RESULTS}

The free energy curves, $A(n(r))$, obtained from ab initio MD and PIMD simulations are displayed in Figure 2(a). These represent in our opinion the most reliable results in the present study. The following features become clear when comparing the results presented in Figure 2(a). The ab initio PIMD results in a much lower free energy for the dissociation process when compared to that of ab initio MD. This effect can be explained by the NQEs, which is expected to lead to lower free energies of dissociation due to the delocalization of the proton ${ }^{46}$ Comparing the results for the isotopologues $\mathrm{H}_{2} \mathrm{O}$ and $\mathrm{T}_{2} \mathrm{O}$ we find that the free energy curves are different in the ab initio PIMD. The calculation of these two species using ab initio MD would result in the same curves due to the absence of NQEs in classical MD . An important consequence of this difference is that the cut off distance $R_{c}$ determined by ab initio MD $(1.30 \pm 0.03 \AA)$ must be adapted to that by ab initio PIMD $\left(1.50 \pm 0.02 \AA\left(\mathrm{H}_{2} \mathrm{O}\right)\right.$ $1.53 \pm 0.04 \AA\left(\mathrm{D}_{2} \mathrm{O}\right)$, see Table II or SI, respectively) in order to reproduce the reference $\mathrm{p} K_{W}$ value of liquid water. We note in passing that the result obtained from ab initio MD is consistent with values used in previous studies of $1.22-1.3 \AA{ }^{14}[17$ One way of interpreting the increase in cut off distance in the PIMD simulations is that the proton stays bonded to the restrained oxygen longer due to NQEs. 
In Figures 2(b) and 2(c) we display the results obtained from semiempirical MD/PIMD and empirical MD/PIMD, respectively. Comparing Figures 2(b) and 2(c) with Figure2(a), the free energy curves of semiempirical MD and PIMD look very different from that of ab initio MD and PIMD in the absolute values. Accordingly, the cutoff distances $R_{c}$ are quite different from one another. On the other hand, the reduction of the free energy curves behaves similarly with respect to the NQEs. Therefore, we speculate that the isotope effects predicted by empirical and semiempirical PIMD can be as reliable as those from ab initio PIMD.

The free energy curves of the classical simulation show an anharmonic behavior since the mean force upon proton dissociation is a nonlinear function of $r$. In addition, the difference between the free energy curves of the classical and quantum simulations vary along $r$, which implies the influence of anharmonicity on the NQEs. In general, the magnitude of the NQEs tends to be large where the potential curvature $\omega$ is larger than $1 / \beta \hbar$, which can be understood from the formula of the quantum harmonic correction to the free energy, $A_{\text {qhc }}=-\beta^{-1} \log \left\{\frac{\beta \hbar \omega / 2}{\sinh (\beta \hbar \omega / 2)}\right\}$. ${ }^{68}$

The computational effort needed to obtain the free energy curve increases proportionally with respect to the number of restraints. We therefore setup the ab initio MD and PIMD simulations with a smaller system and number of beads $(N=32$ and $P=12)$ compared to our previous work on the same system without restraints $(N=64$ and $P=16){ }^{699}$ To verify this setup, we checked the size- and bead-dependence of the MD and PIMD simulations using the semiempirical DFTB potential. Figure 3(a) shows the free energy curves obtained from semiempirical PIMD simulation for a larger number of beads with $P=32$, while Figure 3(b) shows the free energy curves obtained from semiempirical MD and PIMD simulation for a larger system size with 64 water molecules. It can be clearly seen that Figures 3(a) and (b) follow the same trend as Figures 2(b), in the sense that the free energy is reduced by the NQEs within the semiempirical simulations. We can therefore expect that the results obtained from ab initio MD and PIMD simulations shown in Figure 2(a) are reasonable with respect to the nuclear quantum and isotope effects on the free energy curves.

In Table II, we display the autoionization constants, $\mathrm{p} K_{W}$, of liquid $\mathrm{D}_{2} \mathrm{O}$ and $\mathrm{T}_{2} \mathrm{O}$ calculated using Equation (17) and the free energy curves obtained from PIMD simulations. The cutoff parameter $R_{c}$ was determined for a particular setup of PIMD simulations such that the $\mathrm{p} K_{W}$ of liquid $\mathrm{H}_{2} \mathrm{O}$ is 14.00 . We see the trend that the $\mathrm{p} K_{W}$ value of liquid $\mathrm{T}_{2} \mathrm{O}$ 
is higher than 14.00 , which is consistent with experimental expectations. ${ }^{46}$ However, the difference between the $\mathrm{p} K_{W}$ values of liquid $\mathrm{H}_{2} \mathrm{O}$ and $\mathrm{D}_{2} \mathrm{O}$ in the ab initio PIMD simulations lies within the error bars. All the PIMD simulations were able to predict that the $\mathrm{p} K_{W}$ value of liquid $\mathrm{T}_{2} \mathrm{O}$ is larger than that of liquid $\mathrm{H}_{2} \mathrm{O}$ with statistical significance. We also tried calculating $\mathrm{p} K_{W}$ using the $\mathrm{D}_{2} \mathrm{O} \mathrm{p} K_{W}$ as a reference, results given in Table SI of the supplementary information (SI), and we found that the results agree well with the ones presented in Table II.

The autoionization constants calculated using the absolute method outlined around Equation (25) are given in Table III. This method makes it possible to compare the $\mathrm{p} K_{W}$ calculated using MD and PIMD simulations directly, as no reference value is required for this method. Comparing the ab initio MD and PIMD results for $\mathrm{H}_{2} \mathrm{O}$ reveals that the NQEs play an important role in determining the correct $\mathrm{p} K_{W}$ value. We note that the values predicted from the semi empirical MD and PIMD simulations are far from the correct value of $\mathrm{p} K_{W}$, see Table SII in the SI, as can be expected from their free energy profiles. The results using the empirical OSS force field are, however, comparable with those of the ab initio method, with a slightly smaller difference between MD and PIMD results. As shown above, the absolute method of ab initio PIMD simulation can correct for the large overestimation of the unbinding energy of a proton from water of ab initio MD simulation. Additionally, the absolute method produces a reasonable result for the isotope effect for all pure isotopologues studied here.

In Table IV, we display the acidity constants, $\mathrm{p} K_{A}$, associated with the reaction,

$$
\mathrm{HXO}(\mathrm{aq}) \rightleftharpoons \mathrm{OX}^{-}(\mathrm{aq})+\mathrm{H}^{+}(\mathrm{aq}),
$$

where $\mathrm{X}=\mathrm{D}$ or $\mathrm{T}$. The values were calculated using Equation (22) and the free energy curves obtained from PIMD simulations.

Experimental values do not exist for the $\mathrm{p} K_{A}$ of HDO and HTO. However, the "rational" $\mathrm{p} K_{A}$ of $\mathrm{H}_{2} \mathrm{O}$, which is $15.74=\mathrm{p} K_{W}+\log \left(c_{W}\right)$, would be a reference. The rational $\mathrm{p} K_{A}$ is obtained by using an activity for the $\mathrm{H}_{2} \mathrm{O}$ molecules when the dissociating water molecule is assumed misleadingly to be distinguishable from the rest of the water molecules in solution. ${ }^{70171}$ It can however serve well as a reference in this case where the HDO and HTO molecules are in fact distinguishable from the solvent molecules.

It is expected that the $\mathrm{p} K_{A}$ of HDO and HTO in water are either similar to or larger 
than the "rational" $\mathrm{p} K_{A}$ of $\mathrm{H}_{2} \mathrm{O}$, assuming that the isotope effect follows the same trend as the case of the $\mathrm{p} K_{W}$ of $\mathrm{H}_{2} \mathrm{O}, \mathrm{D}_{2} \mathrm{O}$ and $\mathrm{T}_{2} \mathrm{O}$.

Here it turned out that the

$\mathrm{p} K_{A}$ value of HDO predicted from empirical PIMD is close to the "rational" $\mathrm{p} K_{A}$ of $\mathrm{H}_{2} \mathrm{O}$, while the predicted $\mathrm{p} K_{A}$ value of HTO is larger than that of $\mathrm{H}_{2} \mathrm{O}$ with statistical significance. For the semiempirical calculations we find that both the $\mathrm{p} K_{A}$ of HDO and HTO are larger than the "rational" $\mathrm{p} K_{A}$ of $\mathrm{H}_{2} \mathrm{O}$. We note that these $\mathrm{p} K_{A}$ values are difficult to measure experimentally, but they are important in determining the concentration of $\mathrm{OD}^{-}$and $\mathrm{OT}^{-}$ ions in liquid water.

\section{CONCLUSION}

In this study we established a first-principles approach to compute the autoionization and acidity constants of water taking account of NQEs by PIMD with CN restraints. The simulations were carried out using different potential energy surfaces, i.e., ab initio DFT, semiempirical DFTB and empirical OSS2 methods.

The findings presented here are in line with previous empirical results $\frac{46}{64}$ The current study does differentiate itself from previous studies, by targeting the autoionization process directly, without any empirical factors to estimate its free energy curves.

It was found that the free energy curve in the proton dissociation obtained from the quantum PIMD simulation is downshifted significantly compared with that obtained from the classical MD simulation, thus showing the importance of the NQEs on the autoionization and acidity constants. The $\mathrm{p} K_{W}$ values of water isotopologues, liquid $\mathrm{D}_{2} \mathrm{O}$ and $\mathrm{T}_{2} \mathrm{O}$, were estimated based on a probabilistic method using shifts in the free energy curves of $\mathrm{D}_{2} \mathrm{O}$ and $\mathrm{T}_{2} \mathrm{O}$ with respect to that of $\mathrm{H}_{2} \mathrm{O}$. The results agree well with experimental values, accounting for the statistical uncertainties of our simulations.

We went on to compute the $\mathrm{p} K_{A}$ values of aqueous HDO and HTO molecules which are difficult to measure experimentally. The results predict that $\mathrm{p} K_{A}$ of aqueous HTO (HDO) is larger than (close to) that of $\mathrm{H}_{2} \mathrm{O}$.

The work presented here opens the possibility for accurate calculations of $\mathrm{p} K_{A}$ for more complex systems, such as small organic molecules in solution. It furthermore makes it possible to to predict the isotope effect on these system by direct calculation. These goals 
and calculating the temperature and pressure dependence of the autoionization constant of water will be a subject of future studies.

We finally note that the probabilistic method requires the reference $\mathrm{p} K_{W}$ value of $\mathrm{H}_{2} \mathrm{O}$ (14.00) while the absolute method does not. For the latter, however, an accurate estimation of absolute $\mathrm{p} K_{A}$ values remains a difficult challenge. In fact the $\mathrm{p} K_{W}$ estimated using the absolute method with the present simulations have very different values to those from the probablistic method, see Tables II and III. This is because the standard free energy curve is strongly dependent on the potential models and the system sizes. These issues should be studied more carefully in the future. It is however clear that the inclusion of the NQEs is important for determining autoionization and acidity constants.

In fact, we do find a difference of $4.5 \pm 0.9 \mathrm{p} K_{A}$ units between the ab initio MD and PIMD results, where the PIMD result is clearly closer to the true value of the $\mathrm{p} K_{W}$ of water. This difference does seem to be in line, to some extent, with the experimental extrapolation of 3 $\mathrm{p} K_{A}$ units which was suggested earlier $\underline{46}$

\section{SUPPLEMENTARY MATERIAL}

See the supplementary material for Tables showing the autoionization constants of water isotopologues calculated by the probabilistic method using the experimental $\mathrm{p} K_{W}$ of $\mathrm{D}_{2} \mathrm{O}$ to calculate $R_{c}$, and the autoionization constants of water isotopologues calculated by the absolute method using the semiempirical DFTB method.

\section{ACKNOWLEDGEMENTS}

This work was completed under the project "Hydrogenomics" in Grant-in-Aid for Scientific Research on Innovative Areas, MEXT, Japan. The computations were mostly run on the supercomputer facilities at Japan Atomic Energy Agency and the Institute for Solid State Physics, The University of Tokyo. M.S. thanks JSPS KAKENHI (18H05519, 18H01693, 18K05208) and MEXT Program for Promoting Research on the Supercomputer Fugaku (Fugaku Battery \& Fuel Cell Project) for financial support. We thank Prof. Nikos Doltsinis

in Universität Münster for his advice on the coordination number constraints. We thank Dr. Alex Malins in JAEA for proofreading the text. 


\section{DATA AVAILABILITY}

The data that support the findings of this study are available within the article and its supplementary material.

\section{REFERENCES}

${ }^{1}$ K. S. Alongi and G. C. Shields, "Chapter 8 - Theoretical Calculations of Acid Dissociation Constants: A Review Article," in Annual Reports in Computational Chemistry (R. A. Wheeler, ed.), vol. 6, pp. 113-138, Elsevier, Jan. 2010.

${ }^{2}$ E. Alexov, E. L. Mehler, N. Baker, A. M. Baptista, Y. Huang, F. Milletti, J. E. Nielsen, D. Farrell, T. Carstensen, M. H. M. Olsson, J. K. Shen, J. Warwicker, S. Williams, and J. M. Word, "Progress in the prediction of pKa values in proteins," Proteins, vol. 79, pp. 3260-3275, Dec. 2011.

${ }^{3}$ J. Ho and M. Coote, "A universal approach for continuum solvent pK a calculations: are we there yet?," Theor. Chem. Acc., 2010.

${ }^{4}$ J. Tomasi, B. Mennucci, and R. Cammi, "Quantum Mechanical Continuum Solvation Models," Chem. Rev., vol. 105, pp. 2999-3094, Aug. 2005.

${ }^{5}$ S. Miertuš, E. Scrocco, and J. Tomasi, "Electrostatic interaction of a solute with a continuum. A direct utilizaion of $\mathrm{AB}$ initio molecular potentials for the prevision of solvent effects," Chem. Phys., vol. 55, pp. 117-129, Feb. 1981.

${ }^{6}$ J. B. Foresman, T. A. Keith, K. B. Wiberg, J. Snoonian, and M. J. Frisch, "Solvent Effects. 5. Influence of Cavity Shape, Truncation of Electrostatics, and Electron Correlation on ab Initio Reaction Field Calculations," J. Phys. Chem., vol. 100, pp. 16098-16104, Jan. 1996.

${ }^{7}$ J. L. Pascual-ahuir, E. Silla, and I. Tuñon, "GEPOL: An improved description of molecular surfaces. III. A new algorithm for the computation of a solvent-excluding surface," $J$. Comput. Chem., vol. 15, no. 10, pp. 1127-1138, 1994.

${ }^{8}$ S. Miertuš and J. Tomasi, "Approximate evaluations of the electrostatic free energy and internal energy changes in solution processes," Chem. Phys., vol. 65, pp. 239-245, Mar. 1982.

${ }^{9}$ M. Cossi, N. Rega, G. Scalmani, and V. Barone, "Energies, structures, and electronic properties of molecules in solution with the C-PCM solvation model," J. Comput. Chem., 
vol. 24, no. 6, pp. 669-681, 2003.

${ }^{10}$ A. Klamt and G. Schüürmann, "COSMO: a new approach to dielectric screening in solvents with explicit expressions for the screening energy and its gradient," J. Chem. Soc., Perkin Trans. 2, pp. 799-805, Jan. 1993.

${ }^{11}$ A. Klamt, "Conductor-like Screening Model for Real Solvents: A New Approach to the Quantitative Calculation of Solvation Phenomena," J. Phys. Chem., vol. 99, pp. 22242235, Feb. 1995.

${ }^{12}$ A. V. Marenich, C. J. Cramer, and D. G. Truhlar, "Universal Solvation Model Based on Solute Electron Density and on a Continuum Model of the Solvent Defined by the Bulk Dielectric Constant and Atomic Surface Tensions," J. Phys. Chem. B, vol. 113, pp. 63786396, May 2009.

${ }^{13}$ I. Soteras, C. Curutchet, A. Bidon-Chanal, M. Orozco, and F. J. Luque, "Extension of the MST model to the IEF formalism: HF and B3lyp parametrizations," J. Mol. Struct. (Theochem), vol. 727, pp. 29-40, Aug. 2005.

${ }^{14}$ N. L. Doltsinis and M. Sprik, "Theoretical pKa estimates for solvated P(OH)5 from coordination constrained Car-Parrinello molecular dynamics," Phys. Chem. Chem. Phys., vol. 5, pp. 2612-2618, June 2003.

${ }^{15}$ M. Schilling and S. Luber, "Determination of pKa Values via ab initio Molecular Dynamics and its Application to Transition Metal-Based Water Oxidation Catalysts," Inorganics, vol. 7, p. 73, June 2019.

${ }^{16}$ Y.-L. Chen, N. L. Doltsinis, R. C. Hider, and D. J. Barlow, "Prediction of Absolute Hydroxyl pKa Values for 3-Hydroxypyridin-4-ones," J. Phys. Chem. Lett., vol. 3, pp. 29802985, Oct. 2012.

${ }^{17}$ J. E. Davies, N. L. Doltsinis, A. J. Kirby, C. D. Roussev, and M. Sprik, "Estimating pKa Values for Pentaoxyphosphoranes," J. Am. Chem. Soc., vol. 124, pp. 6594-6599, June 2002.

${ }^{18}$ N. Sandmann, J. Bachmann, A. Hepp, N. L. Doltsinis, and J. Müller, "Copper(II)mediated base pairing involving the artificial nucleobase 3h-imidazo[4,5-f]quinolin-5-ol," Dalton Trans., vol. 48, pp. 10505-10515, July 2019.

${ }^{19}$ A. K. Tummanapelli and S. Vasudevan, "Dissociation Constants of Weak Acids from ab Initio Molecular Dynamics Using Metadynamics: Influence of the Inductive Effect and Hydrogen Bonding on pKa Values," J. Phys. Chem. B, vol. 118, pp. 13651-13657, Nov. 
2014.

${ }^{20}$ A. K. Tummanapelli and S. Vasudevan, "Ab Initio MD Simulations of the Brønsted Acidity of Glutathione in Aqueous Solutions: Predicting pKa Shifts of the Cysteine Residue," J. Phys. Chem. B, vol. 119, pp. 15353-15358, Dec. 2015.

${ }^{21}$ A. K. Tummanapelli and S. Vasudevan, "Ab Initio Molecular Dynamics Simulations of Amino Acids in Aqueous Solutions: Estimating pKa Values from Metadynamics Sampling," J. Phys. Chem. B, vol. 119, pp. 12249-12255, Sept. 2015.

${ }^{22}$ C. D. Daub and L. Halonen, "Ab Initio Molecular Dynamics Simulations of the Influence of Lithium Bromide Salt on the Deprotonation of Formic Acid in Aqueous Solution," J. Phys. Chem. B, vol. 123, pp. 6823-6829, Aug. 2019.

${ }^{23} \mathrm{~L}$. Xu and M. L. Coote, "Methods To Improve the Calculations of Solvation Model Density Solvation Free Energies and Associated Aqueous pKa Values: Comparison between Choosing an Optimal Theoretical Level, Solute Cavity Scaling, and Using Explicit Solvent Molecules," J. Phys. Chem. A, vol. 123, pp. 7430-7438, Aug. 2019.

${ }^{24}$ C. C. R. Sutton, G. V. Franks, and G. da Silva, "First Principles pKa Calculations on Carboxylic Acids Using the SMD Solvation Model: Effect of Thermodynamic Cycle, Model Chemistry, and Explicit Solvent Molecules," J. Phys. Chem. B, vol. 116, pp. 11999-12006, Oct. 2012 .

${ }^{25} \mathrm{~S}$. Zhang, "A reliable and efficient first principles-based method for predicting pKa values. III. Adding explicit water molecules: Can the theoretical slope be reproduced and pKa values predicted more accurately?," J. Comput. Chem., vol. 33, no. 5, pp. 517-526, 2012. ${ }^{26}$ B. Thapa and H. B. Schlegel, "Calculations of pKa's and Redox Potentials of Nucleobases with Explicit Waters and Polarizable Continuum Solvation," J. Phys. Chem. A, vol. 119, pp. 5134-5144, May 2015.

${ }^{27}$ B. Thapa and H. B. Schlegel, "Density Functional Theory Calculation of pKa's of Thiols in Aqueous Solution Using Explicit Water Molecules and the Polarizable Continuum Model," J. Phys. Chem. A, vol. 120, pp. 5726-5735, July 2016.

${ }^{28}$ N. L. Haworth, Q. Wang, and M. L. Coote, "Modeling Flexible Molecules in Solution: A pKa Case Study," J. Phys. Chem. A, vol. 121, pp. 5217-5225, July 2017.

${ }^{29}$ E. A. Carter, G. Ciccotti, J. T. Hynes, and R. Kapral, "Constrained reaction coordinate dynamics for the simulation of rare events," Chem. Phys. Lett., vol. 156, pp. 472-477, Apr. 1989. 
${ }^{30}$ M. Sprik and G. Ciccotti, "Free energy from constrained molecular dynamics," J. Chem. Phys., vol. 109, pp. 7737-7744, Nov. 1998.

${ }^{31}$ A. V. Bandura and S. N. Lvov, "The Ionization Constant of Water over Wide Ranges of Temperature and Density," J. Phys. Chem. Ref. Data, vol. 35, pp. 15-30, Dec. 2005.

${ }^{32}$ N. Mora-Diez, Y. Egorova, H. Plommer, and P. R. Tremaine, "Theoretical study of deuterium isotope effects on acid-base equilibria under ambient and hydrothermal conditions," RSC Adv., vol. 5, pp. 9097-9109, Jan. 2015.

${ }^{33}$ D. W. Shoesmith and W. Lee, "The ionization constant of heavy water (D2o) in the temperature range 298 to 523 K," Can. J. Chem., vol. 54, pp. 3553-3558, Nov. 1976.

${ }^{34}$ M. Sprik, "Computation of the pK of liquid water using coordination constraints," Chemical Physics, vol. 258, pp. 139-150, Aug. 2000.

${ }^{35}$ E. Perlt, M. v. Domaros, B. Kirchner, R. Ludwig, and F. Weinhold, "Predicting the Ionic Product of Water," Sci Rep, vol. 7, pp. 1-10, Aug. 2017.

${ }^{36}$ M. Štrajbl, G. Hong, and A. Warshel, "Ab Initio QM/MM Simulation with Proper Sampling: "First Principle" Calculations of the Free Energy of the Autodissociation of Water in Aqueous Solution," J. Phys. Chem. B, vol. 106, pp. 13333-13343, Dec. 2002.

${ }^{37}$ R. Wang, V. Carnevale, M. L. Klein, and E. Borguet, "First-Principles Calculation of Water pKa Using the Newly Developed SCAN Functional," J. Phys. Chem. Lett., vol. 11, pp. 54-59, Jan. 2020.

${ }^{38}$ M. Moqadam, A. Lervik, E. Riccardi, V. Venkatraman, B. K. Alsberg, and T. S. v. Erp, "Local initiation conditions for water autoionization," PNAS, vol. 115, pp. E4569-E4576, May 2018.

${ }^{39}$ P. L. Geissler, C. Dellago, D. Chandler, J. Hutter, and M. Parrinello, "Autoionization in Liquid Water," Science, vol. 291, pp. 2121-2124, Mar. 2001.

${ }^{40}$ B. L. Trout and M. Parrinello, "Analysis of the Dissociation of H2o in Water Using FirstPrinciples Molecular Dynamics," J. Phys. Chem. B, vol. 103, pp. 7340-7345, Aug. 1999.

${ }^{41}$ M. Shiga, "Path Integral Simulations," in Reference Module in Chemistry, Molecular Sciences and Chemical Engineering, Elsevier, Jan. 2018.

${ }^{42}$ M. Parrinello and A. Rahman, "Study of an F center in molten KCl," J. Chem. Phys., vol. 80, pp. 860-867, Jan. 1984.

${ }^{43}$ R. W. Hall and B. J. Berne, "Nonergodicity in path integral molecular dynamics," J. Chem. Phys., vol. 81, pp. 3641-3643, Oct. 1984. 
${ }^{44}$ M. Ceriotti, M. Parrinello, T. E. Markland, and D. E. Manolopoulos, "Efficient stochastic thermostatting of path integral molecular dynamics," J. Chem. Phys., vol. 133, p. 124104, Sept. 2010.

${ }^{45}$ M. E. Tuckerman, B. J. Berne, G. J. Martyna, and M. L. Klein, "Efficient molecular dynamics and hybrid Monte Carlo algorithms for path integrals," J. Chem. Phys., vol. 99, pp. 2796-2808, Aug. 1993.

${ }^{46}$ M. Ceriotti, W. Fang, P. G. Kusalik, R. H. McKenzie, A. Michaelides, M. A. Morales, and T. E. Markland, "Nuclear Quantum Effects in Water and Aqueous Systems: Experiment, Theory, and Current Challenges," Chem. Rev., vol. 116, pp. 7529-7550, July 2016.

${ }^{47}$ D. Marx, M. E. Tuckerman, J. Hutter, and M. Parrinello, "The nature of the hydrated excess proton in water," Nature, vol. 397, pp. 601-604, Feb. 1999.

${ }^{48}$ G. Cassone, "Nuclear Quantum Effects Largely Influence Molecular Dissociation and Proton Transfer in Liquid Water under an Electric Field," J. Phys. Chem. Lett., vol. 11, pp. 8983-8988, Nov. 2020.

${ }^{49}$ D. Chandler, Introduction to Modern Statistical Mechanics. Oxford: Oxford University Press, 1987.

${ }^{50}$ M. Shiga, "PIMD," 2020.

${ }^{51}$ S. Nosé, "A unified formulation of the constant temperature molecular dynamics methods," J. Chem. Phys., vol. 81, pp. 511-519, July 1984.

${ }^{52}$ W. G. Hoover, "Canonical dynamics: Equilibrium phase-space distributions," Phys. Rev. A, vol. 31, pp. 1695-1697, Mar. 1985.

${ }^{53}$ G. J. Martyna, M. L. Klein, and M. Tuckerman, "Nosé-Hoover chains: The canonical ensemble via continuous dynamics," J. Chem. Phys., vol. 97, pp. 2635-2643, Aug. 1992.

${ }^{54} \mathrm{G}$. Kresse and J. Hafner, "Ab initio molecular dynamics for liquid metals," Phys. Rev. B, vol. 47, pp. 558-561, Jan. 1993.

${ }^{55}$ G. Kresse and J. Hafner, "Ab initio molecular-dynamics simulation of the liquid-metalamorphous-semiconductor transition in germanium," Phys. Rev. B, vol. 49, pp. 1425114269, May 1994.

${ }^{56}$ G. Kresse and J. Furthmüller, "Efficiency of ab-initio total energy calculations for metals and semiconductors using a plane-wave basis set," Comput. Mater. Sci., vol. 6, pp. 15-50, July 1996. 
${ }^{57}$ J. P. Perdew, K. Burke, and M. Ernzerhof, "Generalized Gradient Approximation Made Simple," Phys. Rev. Lett., vol. 77, pp. 3865-3868, Oct. 1996.

${ }^{58}$ S. Grimme, J. Antony, S. Ehrlich, and H. Krieg, "A consistent and accurate ab initio parametrization of density functional dispersion correction (DFT-D) for the 94 elements H-Pu," J. Chem. Phys., vol. 132, p. 154104, Apr. 2010.

${ }^{59}$ B. Hourahine, B. Aradi, V. Blum, F. Bonafé, A. Buccheri, C. Camacho, C. Cevallos, M. Y. Deshaye, T. Dumitrică, A. Dominguez, S. Ehlert, M. Elstner, T. van der Heide, J. Hermann, S. Irle, J. J. Kranz, C. Köhler, T. Kowalczyk, T. Kubar̆, I. S. Lee, V. Lutsker, R. J. Maurer, S. K. Min, I. Mitchell, C. Negre, T. A. Niehaus, A. M. N. Niklasson, A. J. Page, A. Pecchia, G. Penazzi, M. P. Persson, J. Řezáč, C. G. Sánchez, M. Sternberg, M. Stöhr, F. Stuckenberg, A. Tkatchenko, V. W.-z. Yu, and T. Frauenheim, "DFTB+, a software package for efficient approximate density functional theory based atomistic simulations," J. Chem. Phys., vol. 152, p. 124101, Mar. 2020.

${ }^{60}$ M. Elstner, D. Porezag, G. Jungnickel, J. Elsner, M. Haugk, T. Frauenheim, S. Suhai, and G. Seifert, "Self-consistent-charge density-functional tight-binding method for simulations of complex materials properties," Phys. Rev. B, vol. 58, pp. 7260-7268, Sept. 1998.

${ }^{61}$ M. Gaus, A. Goez, and M. Elstner, "Parametrization and Benchmark of DFTB3 for Organic Molecules," J. Chem. Theory Comput., vol. 9, pp. 338-354, Jan. 2013.

${ }^{62}$ L. Ojamäe, I. Shavitt, and S. J. Singer, "Potential models for simulations of the solvated proton in water," J. Chem. Phys., vol. 109, pp. 5547-5564, Sept. 1998.

${ }^{63}$ J. Sala, E. Guàrdia, and M. Masia, "The polarizable point dipoles method with electrostatic damping: Implementation on a model system," J. Chem. Phys., vol. 133, p. 234101, Dec. 2010.

${ }^{64}$ N. Michaud-Agrawal, E. J. Denning, T. B. Woolf, and O. Beckstein, "MDAnalysis: A toolkit for the analysis of molecular dynamics simulations," J. Comput. Chem., vol. 32, no. 10, pp. 2319-2327, 2011.

${ }^{65}$ R. J. Gowers, M. Linke, J. Barnoud, T. J. E. Reddy, M. N. Melo, S. L. Seyler, J. Domański, D. L. Dotson, S. Buchoux, I. M. Kenney, and O. Beckstein, "MDAnalysis: A Python Package for the Rapid Analysis of Molecular Dynamics Simulations," Proceedings of the 15th Python in Science Conference, pp. 98-105, 2016.

${ }^{66}$ H. Flyvbjerg and H. G. Petersen, "Error estimates on averages of correlated data," J. Chem. Phys., vol. 91, pp. 461-466, July 1989. 
${ }^{67}$ W. Humphrey, A. Dalke, and K. Schulten, "VMD: Visual molecular dynamics," J. Mol. Graph., vol. 14, pp. 33-38, Feb. 1996.

${ }^{68}$ M. Shiga and H. Fujisaki, "A quantum generalization of intrinsic reaction coordinate using path integral centroid coordinates," J. Chem. Phys., vol. 136, p. 184103, May 2012.

${ }^{69}$ M. Machida, K. Kato, and M. Shiga, "Nuclear quantum effects of light and heavy water studied by all-electron first principles path integral simulations," J. Chem. Phys., vol. 148, p. 102324, Dec. 2017.

${ }^{70}$ T. P. Silverstein and S. T. Heller, "pKa Values in the Undergraduate Curriculum: What Is the Real pKa of Water?," J. Chem. Educ., vol. 94, pp. 690-695, June 2017.

${ }^{71}$ E. C. Meister, M. Willeke, W. Angst, A. Togni, and P. Walde, "Confusing Quantitative Descriptions of Brønsted-Lowry Acid-Base Equilibria in Chemistry Textbooks - A Critical Review and Clarifications for Chemical Educators," Helv. Chim. Acta, vol. 97, no. 1, pp. 131,2014 . 


\section{Figure Captions}

Figure 1: (A) Initial structure containing 32 water molcules. The four inserts on the right show the water molecules within $4 \AA$ of the central oxygen molecule during the simulation with a coordination number restraint of (B) 0.98, (C) 0.80, (D) 0.60, (E) 0.31. The distance from the central oxygen to the nearest constrained hydrogen is (B) $1.00 \AA$, (C) 1.16 $\AA$, (D) $1.29 \AA$, (E) $1.47 \AA$. The $\mathrm{O}-\mathrm{H}$ bonds are for all figures drawn if the O-H distance is less than $1.3 \AA$, with the exception of the $\mathrm{O}^{*}-\mathrm{H}$ bond in which case the bonds are drawn for distances up to $1.5 \AA$. The central oxygen is in (B-E) marked with orange, and the teal hydrogen atom is the only hydrogen atom not subject to any constraints during the simulation.

Figure 2: The free energy curves, $A(n(r))$, for $\mathrm{H}_{2} \mathrm{O}$ using $\mathrm{MD}$ (black), $\mathrm{H}_{2} \mathrm{O}$ using PIMD (orange) and $\mathrm{T}_{2} \mathrm{O}$ using PIMD (green). (a) was calcultated using the ab initio potential, while (b) uses the semiempirical potential and (c) uses the empirical potential. For the MD simulations using OSS2, DFTB and DFT we found the values of $R_{c}$ to be $1.39 \pm 0.02 \AA$, $1.13 \pm 0.00 \AA, 1.27 \pm 0.01 \AA$, respectively. See Table II for the corresponding values for the PIMD simulations. The $R_{c}$ values are shown in this figure as vertical lines for $\mathrm{H}_{2} \mathrm{O}$ using MD (black) and PIMD (orange).

Figure 3: The free energy curves of semiempirical simulations for $\mathrm{H}_{2} \mathrm{O}$ and $\mathrm{T}_{2} \mathrm{O}$ in a box containing (a) 32 and (b) 64 molecules. In both figures the black curve represents classical $\mathrm{MD}$ on $\mathrm{H}_{2} \mathrm{O}$, and the corresponding black vertical line is the calculated $R_{c}$ distance for this simulation, (a) $1.13 \pm 0.00 \AA$ and (b) $1.15 \pm 0.00 \AA$, respectively. The orange and green curves represent $\mathrm{H}_{2} \mathrm{O}$ and $\mathrm{T}_{2} \mathrm{O}$ respectively in a PIMD simulation with (a) $\mathrm{P}=32$ or (b) $\mathrm{P}=12$. The orange vertical lines represent the calculated $R_{c}$ of the two $\mathrm{H}_{2} \mathrm{O}$ PIMD simulations, (a) $1.16 \pm 0.00 \AA$ and (b) $1.17 \pm 0.00 \AA$, respectively. 


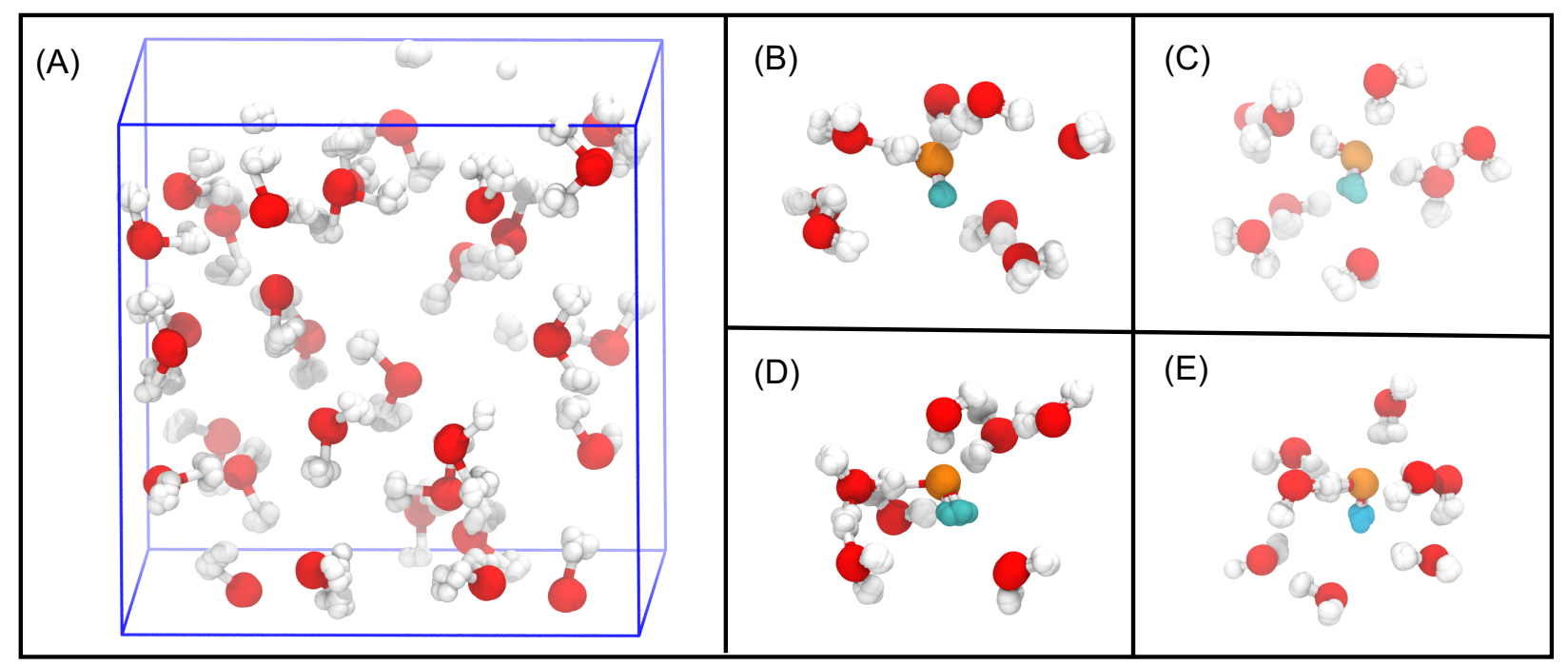

FIG. 1. 


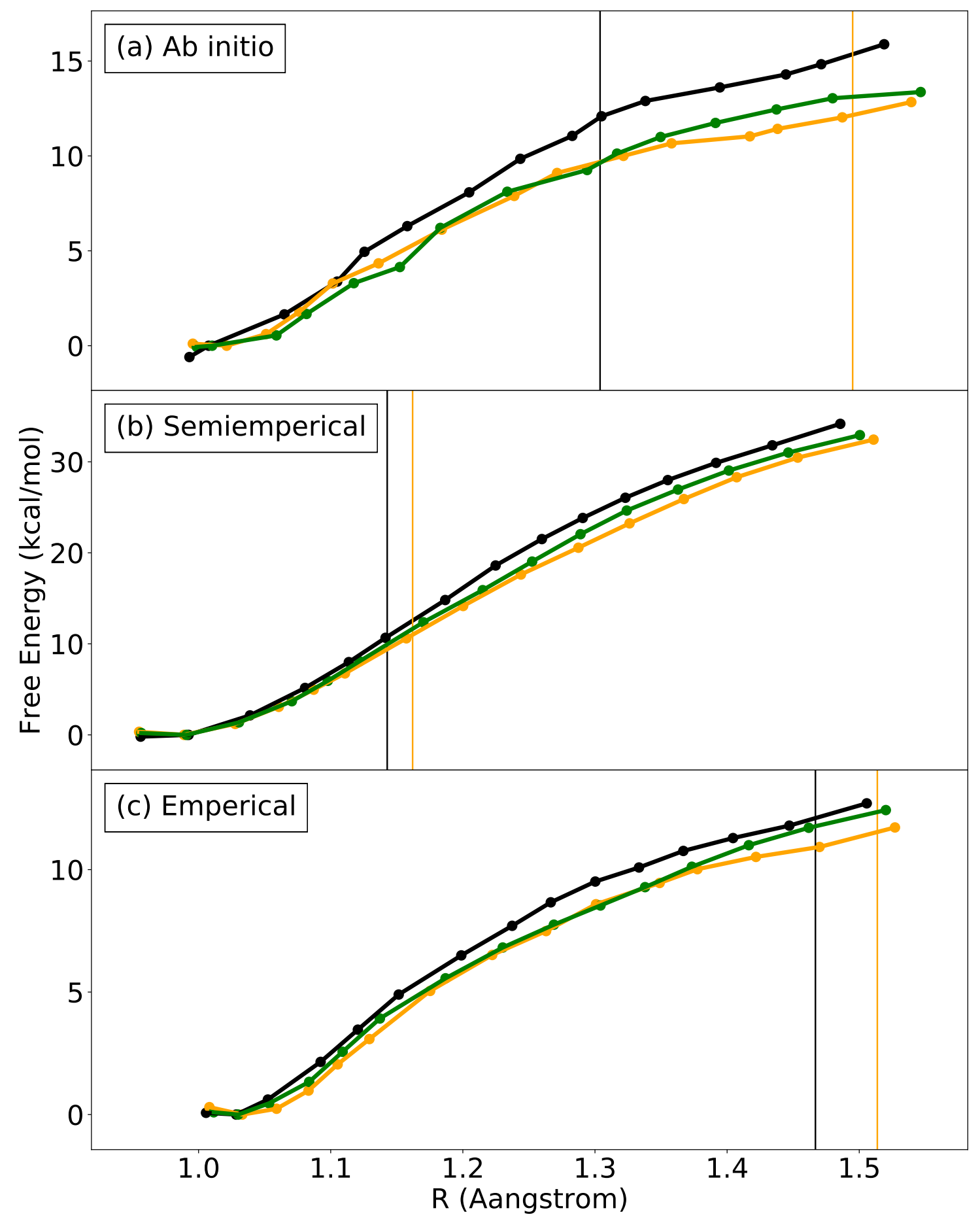

FIG. 2. 


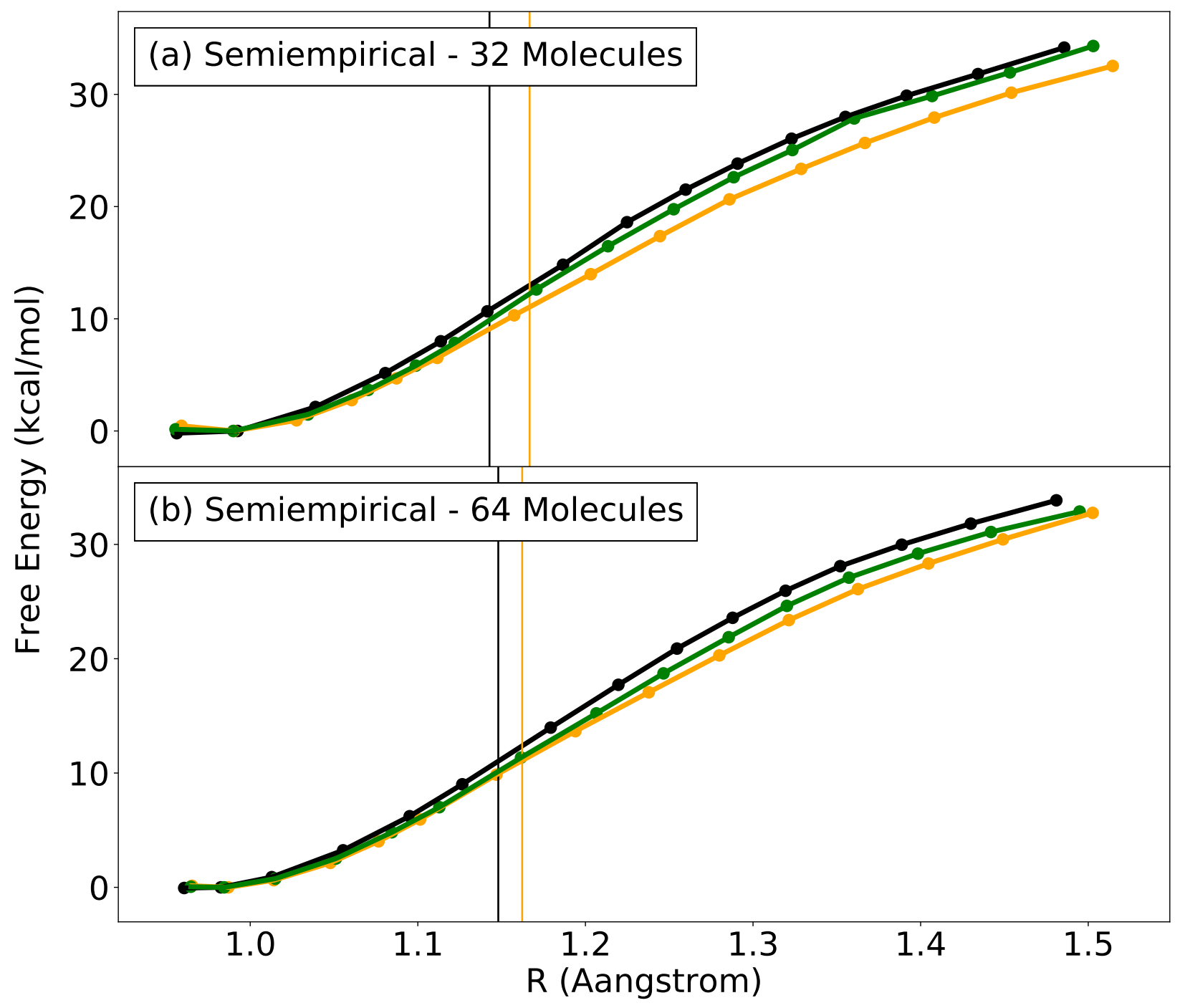

FIG. 3. 
Table I. Simulation setup of water isotopologues.

\begin{tabular}{|c|c|c|c|c|c|c|}
\hline Method & System & Molecules & $P$ & $\Delta t[\mathrm{ps}]$ & Length [ps] & Restraints \\
\hline $\mathrm{DFT}^{a}$ & liq. $\mathrm{H}_{2} \mathrm{O}$ & 32 & 1 & 0.25 & 24.0 & 15 \\
\hline $\mathrm{DFT}^{b}$ & liq. $\mathrm{H}_{2} \mathrm{O}$ & 32 & 12 & 0.25 & 25.0 & 15 \\
\hline $\mathrm{DFT}^{b}$ & liq. $\mathrm{D}_{2} \mathrm{O}$ & 32 & 12 & 0.25 & 25.0 & 15 \\
\hline $\mathrm{DFT}^{b}$ & liq. $\mathrm{T}_{2} \mathrm{O}$ & 32 & 12 & 0.25 & 25.0 & 15 \\
\hline $\mathrm{DFTB}^{c}$ & liq. $\mathrm{H}_{2} \mathrm{O}$ & 32 & 1 & 0.25 & 25.0 & 15 \\
\hline $\mathrm{DFTB}^{d}$ & liq. $\mathrm{H}_{2} \mathrm{O}$ & 32 & 12 & 0.25 & 25.0 & 15 \\
\hline $\mathrm{DFTB}^{d}$ & liq. $\mathrm{H}_{2} \mathrm{O}$ & 32 & 32 & 0.25 & 25.0 & 15 \\
\hline $\mathrm{DFTB}^{d}$ & liq. $\mathrm{D}_{2} \mathrm{O}$ & 32 & 12 & 0.25 & 25.0 & 15 \\
\hline $\operatorname{DFTB}^{d}$ & liq. $\mathrm{T}_{2} \mathrm{O}$ & 32 & 12 & 0.25 & 25.0 & 15 \\
\hline $\mathrm{DFTB}^{d}$ & liq. $\mathrm{T}_{2} \mathrm{O}$ & 32 & 32 & 0.25 & 25.0 & 15 \\
\hline $\mathrm{DFTB}^{d}$ & aq. HDO & 32 & 12 & 0.25 & 25.0 & 15 \\
\hline $\mathrm{DFTB}^{d}$ & aq. HTO & 32 & 12 & 0.25 & 25.0 & 15 \\
\hline $\mathrm{DFTB}^{c}$ & liq. $\mathrm{H}_{2} \mathrm{O}$ & 64 & 1 & 0.25 & 25.0 & 15 \\
\hline $\mathrm{DFTB}^{d}$ & liq. $\mathrm{H}_{2} \mathrm{O}$ & 64 & 12 & 0.25 & 25.0 & 15 \\
\hline $\mathrm{DFTB}^{d}$ & liq. $\mathrm{T}_{2} \mathrm{O}$ & 64 & 12 & 0.25 & 25.0 & 15 \\
\hline OSS2 $2^{e}$ & liq. $\mathrm{H}_{2} \mathrm{O}$ & 64 & 1 & 0.25 & 12.5 & 15 \\
\hline $\mathrm{OSS} 2^{f}$ & liq. $\mathrm{H}_{2} \mathrm{O}$ & 64 & 12 & 0.25 & 12.5 & 15 \\
\hline $\mathrm{OSS} 2^{f}$ & liq. $\mathrm{D}_{2} \mathrm{O}$ & 64 & 12 & 0.35 & 17.5 & 15 \\
\hline $\mathrm{OSS} 2^{f}$ & liq. $\mathrm{T}_{2} \mathrm{O}$ & 64 & 12 & 0.43 & 21.5 & 15 \\
\hline $\mathrm{OSS} 2^{f}$ & aq. HDO & 64 & 12 & 0.25 & 12.5 & 15 \\
\hline $\mathrm{OSS} 2^{f}$ & aq. HTO & 64 & 12 & 0.25 & 12.5 & 15 \\
\hline \multicolumn{7}{|c|}{${ }^{a} \mathrm{Ab}$ initio $\mathrm{MD}$. } \\
\hline \multicolumn{7}{|c|}{${ }^{b} \mathrm{Ab}$ initio PIMD. } \\
\hline \multicolumn{7}{|c|}{${ }^{c}$ Semiempirical MD. } \\
\hline \multicolumn{7}{|c|}{${ }^{d}$ Semiempirical PIMD. } \\
\hline${ }^{e}$ Empiric & al MD. & & & & & \\
\hline Impiri & PIMD. & & & & & \\
\hline
\end{tabular}


Table II. Autoionization constants of water isotopologues calculated by the probabilistic method.

\begin{tabular}{|c|c|c|c|c|c|}
\hline Method & System & Molecules & $P$ & $\mathrm{p} K_{W}(\mathrm{PROB})$ & $R_{c}[\AA]$ \\
\hline $\mathrm{DFT}^{a}$ & liq. $\mathrm{H}_{2} \mathrm{O}$ & 32 & 12 & $14.0^{e}$ & $1.50 \pm 0.02$ \\
\hline $\operatorname{DFT}^{a}$ & liq. $\mathrm{D}_{2} \mathrm{O}$ & 32 & 12 & $13.5 \pm 1.2$ & $1.50 \pm 0.02$ \\
\hline $\mathrm{DFT}^{a}$ & liq. $\mathrm{T}_{2} \mathrm{O}$ & 32 & 12 & $15.4 \pm 0.9$ & $1.50 \pm 0.02$ \\
\hline DFTB $^{b}$ & liq. $\mathrm{H}_{2} \mathrm{O}$ & 32 & 12 & $14.0^{e}$ & $1.16 \pm 0.00$ \\
\hline $\mathrm{DFTB}^{b}$ & liq. $\mathrm{D}_{2} \mathrm{O}$ & 32 & 12 & $15.0 \pm 0.3$ & $1.16 \pm 0.00$ \\
\hline DFTB $^{b}$ & liq. $\mathrm{T}_{2} \mathrm{O}$ & 32 & 12 & $15.0 \pm 0.2$ & $1.16 \pm 0.00$ \\
\hline DFTB $^{b}$ & liq. $\mathrm{H}_{2} \mathrm{O}$ & 32 & 32 & $14.0^{e}$ & $1.17 \pm 0.00$ \\
\hline DFTB $^{b}$ & liq. $\mathrm{T}_{2} \mathrm{O}$ & 32 & 32 & $15.9 \pm 0.3$ & $1.17 \pm 0.00$ \\
\hline DFTB $^{b}$ & liq. $\mathrm{H}_{2} \mathrm{O}$ & 64 & 12 & $14.0^{e}$ & $1.16 \pm 0.00$ \\
\hline DFTB $^{b}$ & liq. $\mathrm{T}_{2} \mathrm{O}$ & 64 & 12 & $14.4 \pm 0.2$ & $1.16 \pm 0.00$ \\
\hline $\mathrm{OSS} 2^{c}$ & liq. $\mathrm{H}_{2} \mathrm{O}$ & 64 & 12 & $14.0^{e}$ & $1.51 \pm 0.01$ \\
\hline OSS $2^{c}$ & liq. $\mathrm{D}_{2} \mathrm{O}$ & 64 & 12 & $15.3 \pm 0.5$ & $1.51 \pm 0.01$ \\
\hline OSS2 ${ }^{c}$ & liq. $\mathrm{T}_{2} \mathrm{O}$ & 64 & 12 & $15.6 \pm 0.5$ & $1.51 \pm 0.01$ \\
\hline $\operatorname{Exptl}^{d}$ & liq. $\mathrm{H}_{2} \mathrm{O}$ & 14.00 & & & \\
\hline $\operatorname{Exptl}^{d}$ & liq. $\mathrm{D}_{2} \mathrm{O}$ & $14.86^{33}$ & & & \\
\hline $\operatorname{Exptl}^{d}$ & liq. $\mathrm{T}_{2} \mathrm{O}$ & $15.2^{46}$ & & & \\
\hline
\end{tabular}

${ }^{a} \mathrm{Ab}$ initio PIMD.

${ }^{b}$ Semiempirical PIMD.

${ }^{c}$ Empirical PIMD.

${ }^{d}$ Experimental values.

${ }^{e}$ Reference value to determine $R_{c}$. 
Table III. Autoionization constants of water isotopologues calculated by the absolute method.

\begin{tabular}{ccccc}
\hline \hline Method & System & Molecules & $P$ & $\mathrm{p} K_{W}(\mathrm{ABS})$ \\
\hline DFT $^{a}$ & liq. $\mathrm{H}_{2} \mathrm{O}$ & 32 & 1 & $19.7 \pm 0.3$ \\
DFT $^{b}$ & liq. $\mathrm{H}_{2} \mathrm{O}$ & 32 & 12 & $15.2 \pm 0.9$ \\
DFT $^{b}$ & liq. $\mathrm{D}_{2} \mathrm{O}$ & 32 & 12 & $14.8 \pm 0.7$ \\
DFT $^{b}$ & liq. $\mathrm{T}_{2} \mathrm{O}$ & 32 & 12 & $16.0 \pm 0.8$ \\
& & & & \\
OSS2 $^{c}$ & liq. $\mathrm{H}_{2} \mathrm{O}$ & 64 & 1 & $15.0 \pm 0.5$ \\
OSS2 $^{d}$ & liq. $\mathrm{H}_{2} \mathrm{O}$ & 64 & 12 & $13.5 \pm 0.5$ \\
OSS2 $^{d}$ & liq. $\mathrm{D}_{2} \mathrm{O}$ & 64 & 12 & $14.9 \pm 0.7$ \\
OSS2 $^{d}$ & liq. $\mathrm{T}_{2} \mathrm{O}$ & 64 & 12 & $14.6 \pm 0.6$ \\
\hline
\end{tabular}

${ }^{a} \mathrm{Ab}$ initio MD.

${ }^{b} \mathrm{Ab}$ initio PIMD.

${ }^{c}$ Empirical MD.

${ }^{d}$ Empirical PIMD. 
Table IV. Acidity constants of water isotopologues, calculated using Equation (23).

\begin{tabular}{cccc}
\hline \hline Method & System & $\mathrm{p} K_{A}(\mathrm{PROP})$ & $R_{c}[\AA]$ \\
\hline DFTB $^{a}$ & aq. HDO & $16.1 \pm 0.1$ & $1.16 \pm 0.00$ \\
DFTB $^{a}$ & aq. HTO & $16.3 \pm 0.1$ & $1.16 \pm 0.00$ \\
$\mathrm{OSS2}^{b}$ & aq. HDO & $15.6 \pm 0.3$ & $1.51 \pm 0.01$ \\
$\mathrm{OSS2}^{b}$ & aq. HTO & $17.1 \pm 0.4$ & $1.51 \pm 0.01$ \\
\hline
\end{tabular}

${ }^{a}$ Semiempirical PIMD of 32 water molecules with $P=12$.

${ }^{b}$ Empirical PIMD of 64 water molecules with $P=12$. 